\title{
How to Choose the Best Option to Treat People with Recurrent Graves' Hyperthyroidism
}

\author{
Garcia-Mayor RV* \\ Endocrine Area, South Galicia Health Research Institute, \\ Spain \\ *Correspondiing author: Ricardo V García-Mayor, \\ South Galicia Health Research Institute, University \\ Hospital of Vigo, Vigo, Spain
}

Received: March 22, 2021; Accepted: April 10, 2021;

Published: April 17, 2021

\section{Editorial}

Graves' Hyperthyroidism (GH) is an autoimmune disease that as other autoimmune diseases is characterized for oscillate activity over its course, thus the patients can enter into a remission phase without undergoing any type of specific treatment. GH is the most common form of hyperthyroidism in populations with an adequate iodine intake, most often affecting women age between 30 to 50 . Untreated $\mathrm{GH}$ is associated with increased mortality and morbidity for this reason timely treatment is critical.

Actually, there is consensus that Antithyroid Drugs (ATD) mainly methimazole are the first option to treat incident cases with GH, being Radioactive Iodine (RI) and a Total Thyroidectomy (TT) reserved as second-line therapies [1]. The major drawback of ATD treatment is its around 50\% recurrence rate of hyperthyroidism after discontinuation of the 12-18 month treatment cycle [2]. However, there is no consensus on the optimal treatment for patients with recurrent $\mathrm{GH}$.

The main therapeutic protocols recommended an open discussion between physician and patients as the best approach to choose the optimal therapy for recurrent GH [3]. The matter of such discussion should consider the advantages and disadvantages for each therapeutical option for the individual patient.

Advantages and disadvantages for each therapeutical modalities:

Advantages of the ATD, preservation of the thyroid tissue, simple administration, low cost, relatively fast control of the hyperthyroidism, good security profile for long-term treatment, side-effects are dose-dependent [4], second ATD cycle in patients with recurrent GH leads to satisfactory long-term remission [5]. RI, definitive hyperthyroidism solution, easy to administer, relatively low cost, relative fast control of the hyperthyroidism. TT, rapid and definitive resolution of the hyperthyroidism.

Disadvantages of the ATD, high relapse rate [6], minor adverse effects, mainly skin reactions $5 \%$ and arthralgia $2 \%$ [4]. RI, athyreotic hypothyroidism no easy to control for life, progression of Graves' orbitopathy, increase overall mortality [7]. TT, athyreotic hypothyroidism treated for life, high dependence of surgeon qualification, transient or permanent recurrent nerve injury and hypoparathyroidism and postoperative bleeding, and high cost.

\section{Conclusion}

In conclusion, taking into account the above mentioned aspects, it appears that treatment with low doses of methimazole would also be the first treatment option for recurrent Graves' hyperthyroidism, being the main indication for RI therapy the patient's preference, and the indication for TT, hyperthyroidism with large and compressive goiter, or with malignant nodules.

\section{References}

1. Bartalena L, Burch HB, Burman KD, Kahaly GJ. A 2013 survey of clinical practice patterns in the management of Graves' Disease. Clin Endocrinol (Oxf.). 2016; 84: 115-120.

2. Abraham P, Avenell A, McGeoch SC, Clark LF, Bevan JS. Antithyroid drug regimen for treating Graves' hyperthyroidism. Cochrane Database Syst Rev. 2010; 2010: CD003420

3. Burch HB, Burman KD, Cooper DS. A 2011 survey of clinicalpractice patterns in the management of Graves' disease. J Clin Endocrinol Metab. 2012; 97: 4549-4558.

4. Garcia-Mayor RV, Larrañaga A. Treatment of Graves' hyperthyroidism with thionamide-derived drugs: Review. Medicinal Chemistry. 2010; 6: 239-246.

5. Liu X, Qiang W, Liu X, Liu L, Liu S, Gao A, et al. A second course of antithyroid drug therapy for recurrent Graves' disease: an experience in endocrine practice. Eur J Endocrinol. 2015; 172: 321-326.

6. Garcia-Mayor RV, Alvarez Vázquez P, Fluiters E, Valverde D. Long term remission after antithyroid drug withdrawal in patients with Graves' hyperthyroidism: parameters with prognostic value. Endocrine. 2019; 63: 316-322.

7. Essi R, Saara M, Heini H, Matti V, Anssi A, Pia J. Cardiovascular morbidity and mortality after treatment of hyperthyroidism with either radioactive iodine or thyroidectomy. Thyroid. 2018; 28: 1111-1120.
Annals Thyroid Res - Volume 7 Issue 2 - 2021

Submit your Manuscript | www.austinpublishing group.com

Garcia-Mayor. () All rights are reserved 